\title{
Best Practices in Workplace Mental Health: An Area for Expanded Research
}

\author{
- \\ STAKEHOLDER RESPONSE \\ Barbara Everett, $\mathrm{PHD}$ \\ Canadian Mental Health Association (CMHA), Ontario
}

$\propto$

\begin{abstract}
Mental health, mental illness and the workplace is a timely topic. Many Canadian employers are recognizing the consequences for their bottom line of not addressing the issue. Dr. Stuart's paper was a thorough discussion of the topic of stigma. I suggested four points for consideration. First, the time may have come to substitute the word discrimination for stigma. It opens a rights and responsibilities dialogue that would be valuable. Also, employers and employees understand the term. Second, there are two populations to consider; those who want to enter the workforce, possibly for the first time, and those who want to stay in the workforce. Studying both populations' needs and experiences would yield new knowledge. Third, consider broadening the investigation scope to include instances where things are working (best practices). And finally, concentration only on anti-stigma programs would exclude other innovations.
\end{abstract}

I AM ALWAYS INTERESTED IN THE question "Why now?" Why now is there a growing interest in mental illness in the workplace when this issue has been with us for many, many decades? One speculative answer to this complex question is the lingering 
aftermath of September 11. At CMHA, Ontario, we have noted an exponential increase in calls from journalists and business people regarding mental illness since the tragedy. It seems that these two powerful groups have developed a sense of personal vulnerability that they hadn't had before - a vulnerability that relates to the fragility of their own mental health. Mental illness has moved from being viewed as an affliction suffered by unfortunate others to something that could affect anyone.

Dr. Stuart's paper is a timely discussion of the issue of mental illness in the workplace. A close reading of her work raises the following questions and comments - which I hope will add to Dr. Stuart's well-crafted discussion:

\section{(1) Perhaps the time has come to} substitute the word discrimination for stigma. Stigma inadvertently bolsters the view of mental illness as occurring to afflicted others rather than something that can happen to me or my loved ones. Antistigma campaigns often rely on messages that entreat so-called "normal" people to be nice to those who are less fortunate than they are. Discrimination, on the other hand, opens up a different dialogue, one based on rights and responsibilities. If Dr. Stuart's research priorities were re-written substituting the word discrimination for stigma, the questions, to my mind, would be substantively changed. Priority 1 : To gain a better understanding of mentalhealth-related discrimination in Canadian work settings. Priority 2: To develop a body of Canadian evidence based on secondary analyses of workplace discrimination to support best-practice solutions for antidiscrimination programs in the workplace/ Priority 3: To develop business-research alliances to support intervention studies of anti-discrimination programs. How research questions are asked profoundly affects the answers produced.

(2) Dr. Stuart offers the caveat that her discussion is focused on the seriously mentally ill. Of necessity (a necessity I would support), she goes on to offer many comments and research findings related to the experiences of people who would be considered mildly mentally ill. In the case of this discussion paper, there are legitimate reasons to define and consider two groups of people and two sets of experiences when discussing mental illness in the workplace. While there is inevitably some overlap between these two groups, there are distinct employment issues. People who have been seriously mentally ill since their late teens have typically had their developmental goals and schooling interrupted and may have never worked in their lives. Their desire to work is nonetheless well proven through research. They have particular needs that relate to the unfair uphill battle they must wage just to enter the workplace in the first place. Then there is a second set of people who have worked and who continue to work, but who struggle with a mental illness that their employer may or may not be aware of. This group wants to keep their employment and climb the career ladder without fear of discriminatory treatment. Put simply, one group wants in, and the other wants to stay in. This distinction would focus the research priorities in ways that could produce relevant and, hopefully, practical findings regarding employment strategies for both groups and their current and potential employers.

(3) Dr. Stuart presents ample evidence from other jurisdictions that discrimina- 
tion in the workplace exists for people with mental illness. However, a somewhat different reading of the statistics raises a different question: What are employers and employees doing when things work out? For example, half of employers surveyed expressed discomfort in hiring someone with a previous hospitalization but half did not. One in three employees reported being counselled to take jobs below their education level, but two out of three did not. I recognize that it is speculative to interpret these statistics as exactly oppositional to one another but, nonetheless, identifying pockets of activity among employers and employees where there may be something positive going on is worthwhile. Therefore, I would add a nuance to all three research priorities suggested by Dr. Stuart, and that is to uncover instances and best practices where people with mental illness are getting and keeping jobs through positive non-discriminatory interactions with employers.

(4) I WONDER IF EXPANDING ALL three research priorities beyond investigating anti-discrimination (anti-stigma) programs in the workplace might net broader findings. Anti-anything campaigns are notoriously ineffective, and there could be a variety of innovative practices to be found in Canadian workplaces that could prove valuable and replicable. 